\title{
Lisières de l'autofiction. Enjeux géographiques, artistiques et politiques, éds. Arnaud Genon \& Isabelle Grell
}

\section{Gabriella Bosco}

\section{(2) OpenEdition}

\section{Journals}

\section{Edizione digitale}

URL: http://journals.openedition.org/studifrancesi/5481

DOI: 10.4000/studifrancesi.5481

ISSN: 2421-5856

\section{Editore}

Rosenberg \& Sellier

\section{Edizione cartacea}

Data di pubblicazione: 1 dicembre 2016

Paginazione: 586-587

ISSN: 0039-2944

\section{Notizia bibliografica digitale}

Gabriella Bosco, «Lisières de l'autofiction. Enjeux géographiques, artistiques et politiques, éds. Arnaud Genon \& Isabelle Grell », Studi Francesi [Online], 180 (LX | III) | 2016, online dal 01 janvier 2017, consultato il 18 septembre 2020. URL : http://journals.openedition.org/studifrancesi/5481; DOI : https://doi.org/10.4000/studifrancesi.5481

\section{Questo documento è stato generato automaticamente il 18 settembre 2020.}

\section{cc) $($ ) $\ominus$}

Studi Francesi è distribuita con Licenza Creative Commons Attribuzione - Non commerciale - Non opere derivate 4.0 Internazionale. 


\title{
Lisières de l'autofiction. Enjeux géographiques, artistiques et politiques, éds. Arnaud Genon \& Isabelle Grell
}

\author{
Gabriella Bosco
}

\section{NOTIZIA}

Lisières de l'autofiction. Enjeux géographiques, artistiques et politiques, Colloque de Cérisy, sous la direction d'Arnaud GENON \& Isabelle GRELL, Lyon, Presses Universitaires de Lyon, 2016, $390 \mathrm{pp}$.

1 Dopo la decade organizzata a Cérisy-la-Salle da Isabelle Grell e Claude Burgelin nel 2008, intitolata Autofiction(s) e pensata come prima proposta di riflessione teorica a tutto tondo trent'anni dopo l'invenzione del termine da parte di Serge Doubrovsky, un nuovo convegno ha riunito nello stesso luogo nel luglio del 2012 gli specialisti della nozione per la presa d'atto di un suo ulteriore evolvere: quello ai margini, il suo valicare frontiere già porose in partenza e via via sempre meno marcate, il suo progressivo espandersi in zone limitrofe, il suo contaminare generi e ambiti ancora diversi. A curare l'incontro, e poi il volume che ne raccoglie gli atti, la stessa Isabelle GRELL, con la collaborazione questa volta di Arnaud GENON, e anche questa volta nella collana che già accolse gli atti della decade del 2008, «A(etc.)» delle Presses Universitaires de Lyon diretta da Roger-Yves Roche, consacrata interamente alle scritture autofictionnelles. L'uso del plurale è d'obbligo, per la natura stessa delle scritture in questione, come riconferma questo volume, articolato su più piani.

Quattro i dossiers che organizzano i numerosi interventi. Il primo, "Sans frontières», esamina le particolarità, le eterogeneità ma anche le affinità tra le più varie scritture dell'io nel mondo: il Giappone, la Francia, l'Africa del Nord, l'Africa del Sud, il Brasile. Il secondo, «Se donner à voir», raccoglie i contributi consacrati a pittura, fotografia, performances, installazioni, cinema. Segue «Du je au nous», che analizza il ruolo della 
scrittura autofictionnelle nella percezione e nella concezione del ruolo dell'intellettuale (scrittore o artista) nel mondo odierno. Infine «Théorie, pratique», sezione in cui viene preso in considerazione il passaggio dall'astrazione teorica alla creazione, tramite il confronto tra una disegnatrice e quattro autori che forniscono il loro punto di vista sulle ricadute pratiche del loro lavoro.

3 Ad aprire i lavori Philippe FOREST, già protagonista nel convegno del 2008, romanziere $\mathrm{e}$ teorico delle scritture in prima persona, com'è noto grande conoscitore della letteratura giapponese cui è dedicato il suo intervento, "Watakushi shôsetsu» et autofiction: quelques notes en marge d'un texte fameux de Kobayashi Hideo (pp. 17-31), nel quale illustra come autori moderni quali Kenzaburo Oé o Tsushima, pur ispirandosi a una forma ormai convenzionale di scrittura personale, il watakushi shôsetsu, vi si siano ufficialmente opposti inventando una forma più complessa e più potente di romanzo dell'io, scrittura che prende le mosse da una costruzione estetica altamente ricercata. Najet LIMAM-TNANI, in Double culture et autofiction chez Marguerite Duras, Assia Djebar, Taos Amrouche et Nina Bouraoui (pp.33-53), s'interroga sullo strappo culturale presente in scritture femminili in prima persona che si oppongono all'alternativa imposta dalla società tra l'adeguamento a forme tradizionali o l'accettazione di quelle della quotidianità, inventandone di dissolte, non sottomesse e profondamente giuste. Mhamed DHAI (Réflexions sur le statut de l'autofiction dans la littérature arabe, pp. 55-71) illustra la diffusione del genere autofictionnel in Marocco e nel mondo arabo in generale. Karen FERREIRA-MEYERS tenta analogo percorso nell'ambito della letteratura africana non francofona, occupandosi di scritture femminili (L'autofiction africaine: du collectif à l'individuel à travers la construction mémorielle, pp. 73-95). Chiude poi la prima sezione la giornalista e scrittrice Luciana HIDALGO che si occupa di autofiction brasiliana (L'autofiction brésilienne: une écriture limite, pp.97-116), dimostrando come essa, ben lungi da forme di autoincensamento soddisfatto, sia invece una scrittura dolorosa e armata.

La seconda sezione, che si interroga sul posto riservato dagli autori a un je visibile, messo in mostra, smontato, costruito e decostruito, si apre con l'intervento di Véronique MONTÉMONT (Des saisissements: photographie, autobiographie, fiction, pp. 119-139) che studia il ruolo effettivamente attribuito alla fotografia inserita da certi autori in «zone contigue della cartografia letteraria», l'autobiografia e l'autofiction. Sandrine MORSILLO, che si occupa di artisti come Christian Boltanski, Gérard Gasiorowski, Josef Beuys e Jean Le Gac, riflette sulla nozione di autorappresentazione (Autofiction et expofiction: entre les deux, l'artiste s'expose, pp.141-159). Parallelamente, Annie PIBAROT studia l'autoritratto praticato da Montaigne come da Cardano, Michel Leiris, François Nourissier, François Bon o Thomas Clerc (Autoportrait et autofiction, pp. 161-177). Seguono due contributi che affrontano la rappresentazione di sé al cinema, di Pierre ARBUS e di Jean-Pierre BOULÉ che analizzano entrambi l'opera filmica di Hervé Guibert, La Pudeur ou l'Impudeur, ciascuno mettendola a confronto con l'opera di un altro autore, Monteiro il primo, Zabat il secondo (L'imaginaire des corps en dé-réalisation dans les films de Monteiro et de Guibert, pp.179-195; La mise en fiction de l'image: Guibert et Zabat, pp. 197-218).

5 La terza sezione affronta poi la dimensione politica delle scritture autofinzionali. Chloé DELAUME, convinta dell'inestricabilità di autoficiton e politica, illustra le poste in gioco, per lei, di una scrittura che si fa tramite del rifiuto di piegare la propria identità a un sistema che vorrebbe rinchiuderla in un mondo prepensato (Politique et autofiction, 
pp.221-234). Arnaud GENON dedica il suo intervento alla scrittura di un autore marocchino, Abdellah Taïa, intellettuale engagé per il quale l'uso del je è di per sé una sfida, in un Paese come il suo in cui scrivere in prima persona è visto come minaccia sociale (L'autofiction comme en(je)u politique dans l'œuvre d'Abdellah Taïa, pp. 235-257). Mentre Nathanaël WADBLED indaga la creazione di un'autofiction filosofica e militante da parte di un'autrice come Judith Butler, che traspone il suo io nel suo pensiero, finzionalizzando quest'ultimo (Dire quelque chose de soi: la théorie du genre de Judith Butler comme autofiction philosophique, pp.259-276). Chiude la sezione Thomas CLERC proponendo una riconfigurazione del campo letterario recente, grosso modo dal 1980 a oggi, a partire dall'opposizione radicale tra autobiografia e autofiction (Le moment sauvage de l'autobiographie: le tournant des années 1990, pp. 277-290).

6 L'ultimo dossier è quello delle esemplificazioni pratiche. La pittrice e scrittrice Anne GOUROUBEN propone illustrazioni del suo trasporre in immagini e in parole i suoi numerosi je (Ni tout à fait la même, ni tout à fait une autre (les autoportraits dans la valise), pp. 293-304). Mathieu SIMONIN, scrittore e avvocato, spiega il suo desiderio di uscire dall'individualismo tramite l'invenzione di un'autobiografia collettiva realizzata mettendo teatralmente in scena il proprio io accanto a quello di un vicino, di uno sconosciuto, di un je altro (Autofiction et jeux collectifs, pp. 305-319). Claire LEGENDRE analizza la propria esperienza vissuta durante un soggiorno a Praga, dimostrando l'impossibilità per uno scrittore ceco di allontanarsi dal principio di verità che fonda l'autobiografia secondo la visione lejeuniana (Une expérience de l'autofiction à Prague: l'écriture transfrontalière (2008-2012)», pp.321-352). La scrittrice québécoise Mélikah ABDELMOUMEN poi esibisce la propria non-scelta dell' autofiction determinata dall'appartenenza a un certo paese, le origini biologiche, il sessismo, l'esclusione in virtù del colore della pelle o del suono di un nome (Mon ennemie préférée: l'autofiction jouée, déjouée, rejouée, pp.353-368). Infine, a chiusura della sezione e del volume, un dialogo tra Abdellah TAÏA e Arnaud Genon (Tout ce que j'écris coule de la même source: moi, pp.369-381) che fa il punto, in maniera di conclusione, su ciò che significa, oggi, assumere una postura autofinzionale, e il contenuto etico di tale postura. L'autofiction oggi, come osserva Isabelle GRELL nelle pagine introduttive (pp.5-13) è la scrittura engagée di un io che testimonia di un essere-nel-mondo assunto nella sua frammentazione, nella sua mondializzazione e nel suo rapporto con gli altri. 